\title{
EXPONENTIAL INTEGRABILITY OF THE QUASI-HYPERBOLIC METRIC ON HÖLDER DOMAINS
}

\author{
Wayne Smith and David A. Stegenga
}

\begin{abstract}
A proper subdomain $D$ of $R^{n}$ is called a Hölder domain if for a fixed $y$ in $D$, the quasi-hyperbolic metric $k_{D}(x, y)$ is bounded by a constant plus a constant multiple of the logarithm of the Euclidean distance from $x$ to the boundary of $D$. For simply connected planar domains $D$, it is known that these domains are characterized by the fact that the Riemann mapping function of the unit disk onto $D$ satisfies a Hölder condition with some positive exponent.

For $y$ in $D$ fixed, we prove that $\exp \left(\tau k_{D}(x, y)\right)$ is integrable over $D$ for some $\tau>0$. One corollary of this is that the boundaries of these domains have Hausdorff dimension less than $n$. Other applications pertain to Poincaré domains and to averaging domains. Our method involves extending some recent results of Carleson-Jones and Jones-Makarov on simply connected planar domains to multiply connected domains in $R^{n}$ by using the quasi-hyperbolic metric.
\end{abstract}

\section{Introduction}

Consider an open, connected and proper subdomain $D$ of Euclidean $n$-space $R^{n}, n \geq 2$. Following [GO] we define the quasi-hyperbolic metric $k_{D}$ in $D$ by

$$
k_{D}\left(x_{1}, x_{2}\right)=\inf _{\gamma} \int_{\gamma} \frac{d s}{\delta_{D}(x)}
$$

where the infimum is taken over all rectifiable arcs $\gamma$ joining $x_{1}$ to $x_{2}$ in $D$. Here we denote by $\delta_{D}(x)$ the Euclidean distance between $x$ and ${ }^{c} D$, the complement of $D$. As usual, we define

$$
k_{D}\left(x_{1}, A\right)=\inf _{y \in A} k_{D}\left(x_{1}, y\right)
$$

for $x_{1} \in D$ and $A \subset D$.

If $D$ is a simply connected planar domain, then the quasi-hyperbolic metric is comparable to the usual hyperbolic or Poincare metric on $D$. See, for example, [BP]. For domains in $R^{n}$, the quasi-hyperbolic metric provides a useful substitute for the hyperbolic metric. Applications can be found, for example, in [GM], [GP], $[\mathrm{GO}],[\mathrm{H}],[\mathrm{S}]$ and $[\mathrm{SS} 2]$.

The first author is supported in part by a grant from the National Science Foundation.

AMS 1991 Subject Classification: Primary 46E35; Secondary 30C55, 30C60, 35Q99, 42B99. 
Definition. Let $D$ be a proper subdomain of $R^{n}$ and let $x_{0} \in D$. The domain $D$ is said to be a Hölder domain if there are constants $c$ and $C$ such that

$$
k_{D}\left(x_{0}, x\right) \leq c \log \frac{\delta_{D}\left(x_{0}\right)}{\delta_{D}(x)}+C, \quad x \in D .
$$

The terminology is derived from the fact that in the plane, a simply connected domain $D$ is a Hölder domain if and only if a Riemann mapping function from the unit disk onto $D$ is Hölder continuous. (See [BP].) In general, Hölder domains are bounded and multiply connected domains.

Our motivation for this paper stems from our interest in the geometry of Hölder domains and the Poincaré inequality. In [SS2] Corollary 4 we showed that the volume of the boundary of a Hölder domain is 0 , and we asked whether the Hausdorff dimension of the boundary must be less than $n$. This was known for John domains by the results in [MV]; see also Theorem 4 in [SS2]. It is a consequence of part (b) in the theorem below that this dimensionality result extends to Hölder domains.

Theorem A. Let $D$ be a proper subdomain of $R^{n}$ and let $x_{0} \in D$. The following are equivalent:

(a) $D$ is a Hölder domain;

(b) There is a $\tau>0$ such that

$$
\int_{D} \exp \left(\tau k_{D}\left(x_{0}, x\right)\right) d x<\infty
$$

(c) $D$ has finite volume, $m(D)<\infty$, and there is a $\tau>0$ such that whenever $u$ is integrable on $D$ and satisfies

$$
\sup _{B} \frac{1}{m(B)} \int_{B} \exp \left(\left|u-u_{B}\right|\right) d x \leq 2
$$

where the supremum is over all balls $B \subset D$ and $u_{B}$ denotes the average of $u$ over $B$, then

$$
\frac{1}{m(D)} \int_{D} \exp \left(\tau\left|u-u_{D}\right|\right) d x \leq 2 .
$$

These equivalences are contained in Theorem 2 and Theorem 4 below. Our main result, Theorem 1, provides an estimate of the number of cubes, in a Whitney decomposition $W$ of a general domain $D$, of a given size among those cubes in $W$ that are at approximately the same quasi-hyperbolic distance from a fixed point $x_{0} \in D$. We use this estimate together with geometric conditions on a domain to 
derive integrability properties of the quasi-hyperbolic metric. The applications to Hölder domains are given in Theorem 2 and Theorem 4. A precise statement of Theorem 1 and its proof can be found in Section 2. Theorem 1 extends some recent results of Carleson and Jones, see Section 10 of [CJ], and Jones and Makarov [M] who considered simply connected planar domains.

The next applications of Theorem 1 concern the Poincare inequality on a domain in $R^{n}$. Let $D \subset R^{n}$ be a domain with finite volume and $1 \leq p<\infty$. We denote by $W^{1, p}(D)$ the usual Sobolev space of functions on $D$ that together with their first order weak partial derivatives are in $L^{p}(D)$. The norm for $W^{1, p}(D)$ is given by

$$
\|u\|_{W^{1, p}(D)}=\left(\int_{D}|u|^{p} d x+\int_{D}|\nabla u|^{p} d x\right)^{1 / p} .
$$

Define

$$
M_{p}(D)=\sup _{u} \frac{\left(\int_{D}\left|u-u_{D}\right|^{p} d x\right)^{1 / p}}{\left(\int_{D}|\nabla u|^{p} d x\right)^{1 / p}},
$$

where the supremum is taken over all nonconstant $u \in W^{1, p}(D)$ and $u_{D}$ is the average of $u$ over $D . D$ is said to be a $p$-Poincaré domain if $M_{p}(D)<\infty$.

In Theorem 1 [SS2] it was established that if $D$ is a Hölder domain, then $D$ is a $p$-Poincare domain for $p \geq n$ and furthermore that this result is best possible for the class of all Hölder domains. Then Hurri $[\mathrm{H}]$ proved that a Hölder domain satisfying an additional geometric condition is a $p$-Poincare domain for all $p>n-\varepsilon$, where $\varepsilon$ depends on the domain. In Section 3, we use Theorem 1 to show that Hurri's geometric condition holds for all Hölder domains and hence the improved Poincaré inequality holds also. A special case of Theorem 1 [SS2] is that, if $D \subset \mathbf{C}$ is the image of the unit disk in the complex plane under a Hölder continuous Riemann mapping function, then $M_{2}(D)<\infty$. An extension of this result to other Riemann mapping functions is given in Section 3.

The final section of the paper is concerned with averaging domains. An averaging domain is a domain for which local BMO-type norm estimates imply a global BMO-type norm estimate. A precise definition can be found in Section 4. Our work here was motivated by that of Staples [S]. A characterization of averaging domains involving the quasi-hyperbolic metric is given, and then this and Theorem 2 are used to give the characterization of Hölder domains given in part (c) in the above theorem.

Acknowledgment. The authors would like to express their gratitude to Professor N.G. Makarov for generously sharing with us his insights and some of the results from his collaboration with P. Jones. We happily acknowledge the valuable contribution he has made to this paper. We also thank Professor P. Jones for providing us with an early version of his manuscript [CJ]. 


\section{Main result}

Throughout this paper $W=\{Q\}$ will be a Whitney decomposition of $D$ into closed dyadic cubes with disjoint interiors. This means that the coordinates of the vertices of each cube are dyadic rational numbers and that the diameter of each cube $Q \in W$, which we denote by $d(Q)$, is comparable to its Euclidean distance to $\partial D$. Further, the constants of comparability do not depend on $D$. See Chapter 6 of Stein's book [St] for the existence of such a decomposition.

The volume of a measurable subset $E$ of $R^{n}$ will be denoted by $m(E)$. The notation $a \approx b$ and $a \lesssim b$ will be used to mean that $a$ and $b$ are either comparable or satisfy an inequality with a constant depending only on the dimension.

The integral of Marcinkiewicz (associated with the domain $D$ ) is defined by

$$
M(x)=\int_{D} \frac{\delta_{D}(y)}{|x-y|^{n+1}} d y .
$$

See Chapter I of Stein's book [St]. We shall require the following fundamental result concerning these integrals; see Lemma 5 in [C1] or [Z]. We sketch a different proof which emphasizes the independence of the constants on the domain.

Lemma 1. Let $D$ be a domain in $R^{n}$ and let $Q_{0}$ be a cube with $D \subset Q_{0}$. There are positive constants $c_{1}$ and $c_{2}$ depending only on the dimension $n$ such that if $\lambda>0$, then

$$
m\left(\left\{x \in Q_{0} \backslash D \mid M(x)>\lambda\right\}\right)<c_{1} m\left(Q_{0}\right) \exp \left(-c_{2} \lambda\right) .
$$

Proof. We first use the Whitney decomposition of $D$ to define a measure on $R_{+}^{n+1}=\left\{(x, y) \mid x \in R^{n}, y>0\right\}$. For $Q \in W$, let $\mu_{Q}$ be a point mass at $\left(x_{Q}, d(Q)\right)$ of weight $m(Q)$, where $x_{Q}$ is the center of $Q$, and let $\mu_{D}=\sum_{Q \in W} \mu_{Q}$. It is immediate that $\mu_{D}$ is a Carleson measure with norm bounded by a constant independent of $D$; that is, for an arbitrary cube $Q^{\prime} \subset R^{n}, \mu\left(Q^{\prime} \times d\left(Q^{\prime}\right)\right) \leq$ $c \cdot m\left(Q^{\prime}\right)$. It follows that the sweep of $\mu, S_{\mu}(x)=\int P_{y}(x-t) d \mu(t, y)$ where $P_{y}(t)$ is the Poisson kernel on $R^{n}$, is a function of bounded mean oscillation with norm less than an absolute constant. See [C2] or Chapter 6 in [G]. Furthermore, an easy computation shows that for $x \in{ }^{c} D, M(x) \approx S_{\mu}(x)$. The result is now a consequence of the John-Nirenberg Theorem [JN] (or see Chapter 6 in [G]) applied to the restriction of $S_{\mu}$ to $Q_{0}$.

For $r>0$ and $x \in R^{n}$, let $T(r ; x)=\{y \in D|| x-y \mid=r\}$, and define $\rho(r ; x)=\max \left\{\delta_{D}(y) \mid y \in T(r ; x)\right\}$.

Lemma 2. Let $x_{0} \in D$ and make the normalizing assumption that $\delta_{D}\left(x_{0}\right)$ $=1$. Suppose $x \in{ }^{c} D$ is such that the distance from $x$ to $D$ satisfies $0<$ $\operatorname{dist}(x, D)=r_{0}<1$ and let $c$ be a constant satisfying $1<c<1 / r_{0}$. Then

$$
M(x) \gtrsim\left(\log \frac{1}{c r_{0}}\right)^{n+1} \cdot k_{D}^{-n}\left(x_{0}, T\left(c r_{0} ; x\right)\right) .
$$


Proof. We first estimate $M(x)$ by using polar coordinates with center $x$. Let $d \sigma$ be surface measure on the unit sphere $\Omega$ in $R^{n}$.

$$
\begin{aligned}
M(x) & \geq \int_{0}^{1} \int_{\Omega} \frac{\delta_{D}(r s)}{r^{n+1}} r^{n-1} d \sigma(s) d r \\
& \geq \int_{r_{0}}^{1} \frac{1}{r^{2}} \int_{\left\{s \in \Omega \mid \delta_{D}(r s) \geq \rho(r ; x) / 2\right\}} \delta_{D}(r s) d \sigma(s) d r \\
& \gtrsim \int_{r_{0}}^{1} \frac{1}{r^{2}} \rho(r ; x)\left(\frac{\rho(r ; x)}{r}\right)^{n-1} d r \\
& =\int_{r_{0}}^{1} \frac{\rho(r ; x)^{n}}{r^{n+1}} d r .
\end{aligned}
$$

If $\gamma$ is a rectifiable curve in $D$ from $x_{0}$ to $x_{1} \in T\left(c r_{0} ; x\right)$, then

$$
\int_{c r_{0}}^{1} \frac{d r}{\rho(r ; x)} \leq \int_{\gamma} \frac{d s}{\rho(|x-y| ; x)} \leq \int_{\gamma} \frac{d s}{\delta_{D}(y)} .
$$

Consequently,

$$
\int_{c r_{0}}^{1} \frac{d r}{\rho(r ; x)} \leq k_{D}\left(x_{0}, T\left(c r_{0} ; x\right)\right)
$$

Thus by Hölder's inequality, (2.1) and (2.2), we have

$$
\begin{aligned}
\left(\log \frac{1}{c r_{0}}\right)^{n+1} & =\left(\int_{c r_{0}}^{1} \frac{\rho(r ; x)^{n /(n+1)}}{r} \cdot \frac{1}{\rho(r ; x)^{n /(n+1)}} d r\right)^{n+1} \\
& \leq \int_{c r_{0}}^{1} \frac{\rho(r ; x)^{n}}{r^{n+1}} d r \cdot\left(\int_{c r_{0}}^{1} \frac{d r}{\rho(r ; x)}\right)^{n} \\
& \lesssim M(x) \cdot k_{D}^{n}\left(x_{0}, T\left(c r_{0} ; x\right)\right)
\end{aligned}
$$

as required. This completes the proof of the lemma.

By refining the Whitney decomposition $W$ of $D$, we may assume without loss of generality that if $Q \in W$ and $x_{1}, x_{2} \in Q$, then $k_{D}\left(x_{1}, x_{2}\right) \leq 1 / 3$. That is, the quasi-hyperbolic diameter of every Whitney cube is less than $1 / 3$. Fix $x_{0} \in D$, and for each $j \geq 0$, define

$$
D_{j}=\cup\left\{Q \in W \mid k_{D}\left(x_{0}, Q\right) \leq j\right\} .
$$


Let $x_{1} \in D_{j-1}$ where $j \geq 1$. Then $k_{D}\left(x_{0}, x_{1}\right) \leq j-2 / 3$ and it follows that $k_{D}\left(x_{0}, x\right) \leq j-1 / 3$ for all points $x$ within a ball centered at $x_{1}$ of radius $\varepsilon \delta_{D}\left(x_{1}\right)$, where $\varepsilon$ is a positive numerical constant. Thus,

$$
\delta_{D_{j}}(x) \approx \delta_{D}(x), \quad x \in D_{j-1} .
$$

Theorem 1. Let $D$ be bounded with $x_{0} \in D$ and $D_{j}$ as above, and put $\delta_{0}=\delta_{D}\left(x_{0}\right)$. Suppose that $t>0$ and $j \geq 1$ are given. There are positive constants $c_{3}$ and $c_{4}$ depending only on the dimension $n$ such that

$$
\sum_{\substack{d(Q) \leq \delta_{0} \exp (-t j) \\ Q \subset D_{j} \backslash D_{j-1}}} m(Q) \leq c_{3} d(D)^{n} \exp \left(-c_{4} t^{n+1} j\right) .
$$

Remark. We were led to this theorem by similar results, for simply connected planar regions, in [CJ] and $[\mathrm{M}]$.

Proof. By a dilation argument, we may assume that $\delta_{0}=1$. Fix $t>0$ and $j \geq 1$, and suppose $Q \in W$ satisfies $d(Q) \leq \exp (-t j)$ and $Q \subset D_{j} \backslash D_{j-1}$. First assume that $1 \leq j \leq 4$. From Lemma 2.1 in [GP] we have that for some $x \in Q$,

$$
\log \frac{\delta_{0}}{\delta_{D}(x)} \leq k_{D}\left(x_{0}, x\right) \leq j \leq 4
$$

and hence that

$$
e^{-4} \leq \frac{\delta_{D}(x)}{\delta_{0}} \lesssim \frac{d(Q)}{\delta_{0}} \leq e^{-t}
$$

Thus, $t \lesssim 1$ and (2.4) follows.

Now suppose that $j \geq 4$. We may assume that $t j$ is large, for if $t j \lesssim 1$, then $t \lesssim 1$ and (2.4) is trivially true. Fix $x \in Q$ and let $r_{0}=\operatorname{dist}\left(x, D_{j-2}\right)$. Observe that if $Q_{1} \in W$ shares a boundary point with $Q$, then $Q_{1}$ is disjoint from $D_{j-2}$. For otherwise, $k_{D}\left(x_{0}, Q\right) \leq k_{D}\left(x_{0}, Q_{1}\right)+1 / 3<j-1$ which violates the condition that $Q \notin D_{j-1}$. Thus, $r_{0} \gtrsim d(Q)$, since neighboring Whitney cubes are comparable in size. By [GO] Lemma 1, there is a geodesic $\gamma$ for the quasihyperbolic metric on $D$ from $x$ to $x_{0}$. Let $x_{1} \in \gamma$ satisfy $k_{D}\left(x_{0}, x_{1}\right)=j-3$. Then, since $k_{D}\left(x_{0}, x\right) \leq j+1 / 3$, we have

$$
\begin{aligned}
3+\frac{1}{3} & \geq k_{D}\left(x, x_{1}\right)=\int_{\gamma\left(x, x_{1}\right)} \frac{d s}{\delta_{D}(y)} \\
& \geq \int_{\gamma\left(x, x_{1}\right)} \frac{d s}{\delta_{D}(x)+s}=\log \left(\frac{\left|\gamma\left(x, x_{1}\right)\right|+\delta_{D}(x)}{\delta_{D}(x)}\right),
\end{aligned}
$$


where $\gamma\left(x, x_{1}\right)$ is the portion of $\gamma$ from $x$ to $x_{1}$ and $\left|\gamma\left(x, x_{1}\right)\right|$ is its length. Thus we have shown that

$$
\operatorname{dist}\left(x, D_{j-3}\right) \leq\left|\gamma\left(x, x_{1}\right)\right| \lesssim \delta_{D}(x) \lesssim r_{0},
$$

and it follows that $r_{0} \approx d(Q)$.

We now estimate $M_{j-2}(x)$ by using Lemma 2 , where $M_{j-2}$ is the integral of Marcinkiewicz associated with the domain $D_{j-2}$. Let $T_{j-2}\left(c r_{0} ; x\right)=\left\{y \in D_{j-2} \mid\right.$ $\left.|x-y|=c r_{0}\right\}$, where $c \lesssim 1$ has been chosen so that $T_{j-2}\left(c r_{0} ; x\right) \cap D_{j-3}$ is not empty. This is possible by (2.6). Thus, by (2.3),

$$
k_{D_{j-2}}\left(x_{0}, T_{j-2}\left(c r_{0} ; x\right)\right) \approx k_{D}\left(x_{0}, T_{j-2}\left(c r_{0} ; x\right)\right) \leq j-3 .
$$

Since $r_{0} \approx d(Q) \leq \exp (-t j)$ and $t j$ is large we obtain from Lemma 2 that

$$
M_{j-2}(x) \gtrsim\left(\log \frac{1}{c r_{0}}\right)^{n+1} \cdot k_{D_{j-2}}^{-n}\left(x_{0}, T_{j-2}\left(c r_{0} ; x\right)\right) \gtrsim t^{n+1} j .
$$

Applying Lemma 1, with $Q_{0}$ being the smallest cube containing $D$, along with the above estimate for $M_{j-2}$ shows that

$$
\begin{aligned}
\sum_{\substack{d(Q) \leq \exp (-t j) \\
Q \subset D_{j} \backslash D_{j-1}}} m(Q) & \leq m\left(\left\{x \in Q_{0} \backslash D_{j-2} \mid M_{j-2}(x)>c_{0} t^{n+1} j\right\}\right) \\
& \lesssim d(D)^{n} \exp \left(-c_{0} c_{2} t^{n+1} j\right)
\end{aligned}
$$

where the constant $c_{0} \approx 1$ comes from (2.7). Thus, the proof is complete.

\section{Applications to Hölder domains}

In this section several applications of Theorem 1 are given. We begin with applications to Hölder domains. If $0<\alpha \leq 1$ we say that a Hölder domain $D \subset R^{n}$ is an $\alpha$-Hölder domain if there is $x_{0} \in D$ and $C<\infty$ such that

$$
k_{D}\left(x_{0}, x\right) \leq \frac{1}{\alpha} \log \frac{1}{\delta_{D}(x)}+C, \quad x \in D .
$$

The restriction $\alpha \leq 1$ is needed because of the inequality (2.5). This terminology derives from the fact that, for a simply connected proper subdomain $D$ of $R^{2}$, there is a Riemann mapping function from the unit disk onto $D$ that is Hölder continuous with exponent $\alpha$ if and only if (3.1) holds when $k_{D}$ is replaced by the comparable hyperbolic metric [BP]. 
Theorem 2. Let $D$ be a domain in $R^{n}$ and let $x_{0} \in D$. The following are equivalent:

(a) There exists $\alpha>0$ such that $D$ is an $\alpha$-Hölder domain;

(b) There exists $\beta>0$ such that $m\left(\left\{x \in D \mid k_{D}\left(x_{0}, x\right)>j\right\}\right)=O(\exp (-\beta j))$, as $j \rightarrow \infty$;

(c) There exists $\tau>0$ such that $\int_{D} \exp \left(\tau k_{D}\left(x_{0}, x\right)\right) d x<\infty$.

Moreover, the constants $\alpha, \beta$ and $\tau$ are related as follows: If (b) or (c) holds, then both hold with $\beta \approx \tau$; if $D$ is an $\alpha$-Hölder domain, then (b) holds with $\beta \gtrsim \alpha^{n+1}$; if (b) holds with $\beta>0$, then $D$ is a $\beta / n$-Hölder domain.

Remark. It can be shown that if a proper subdomain $D$ of $R^{n}$ satisfies the integrability condition in part (c) above, then necessarily $\tau<1$.

Proof. We assume without loss of generality that $\delta_{D}\left(x_{0}\right)=1$. Suppose first that $D$ is an $\alpha$-Hölder domain with Whitney decomposition $W=\{Q\}$, and let $D_{j}$ be as in Section 2. By (3.1) and the properties of a Whitney decomposition, if $j$ is sufficiently large, then

$$
d(Q) \leq \exp \left(-\frac{\alpha}{2} j\right), \quad Q \subset D_{j} \backslash D_{j-1} .
$$

Together with Theorem 1, this gives that if $j$ is large, then

$$
m\left(D_{j} \backslash D_{j-1}\right)=\sum_{\substack{d(Q) \leq \exp (-\alpha j / 2) \\ Q \subset D_{j} \backslash D_{j-1}}} m(Q) \leq c_{3} d(D)^{n} \exp \left(-c_{4}\left(\frac{\alpha}{2}\right)^{n+1} j\right) .
$$

Hence,

$$
m\left(\left\{x \in D \mid k_{D}\left(x_{0}, x\right)>j\right\}\right) \leq \sum_{i \geq j} m\left(D_{i} \backslash D_{i-1}\right) \leq C \exp (-\beta j),
$$

where $\beta=c_{4}(\alpha / 2)^{n+1}$ and the constant $C$ depends on the domain $D$. Thus (b) holds.

Next suppose that (b) holds with $\beta>0$, and fix $Q \in W$. Then

$$
d(Q)^{n} \leq m\left(\left\{x \in D \mid k_{D}\left(x_{0}, x\right)>k_{D}\left(x_{0}, Q\right)\right\}\right) \leq C \exp \left(-\beta k_{D}\left(x_{0}, Q\right)\right) .
$$

By taking logarithms and using that $d(Q) \approx \delta_{D}(x)$ for $x \in Q$ and that the quasihyperbolic diameter of a Whitney cube is comparable to 1 , it follows that (3.1) holds for $x \in Q$ with $\alpha=\beta / n$ and $C \lesssim 1$. Thus, since $Q \in W$ was arbitrary, $D$ is a $\beta / n$-Hölder domain.

The equivalence of (b) and (c) is elementary and is left to the reader. This completes the proof. 
Corollary 1. Let $D$ be an $\alpha$-Hölder domain with Whitney decomposition $W=\{Q\}$. There is a constant $\beta \gtrsim \alpha^{n+1}$ such that

$$
m\left(\cup\left\{Q \in W \mid d(Q)=2^{-j}\right\}\right)=O\left(2^{-\beta j}\right), \quad \text { as } j \rightarrow \infty .
$$

Remark. A domain satisfying (3.2) is said to satisfy a Whitney-\# condition by Martio and Vuorinen. In [MV], they prove that this condition holds for domains satisfying their $c$-covering condition. However, it is not hard to construct Hölder domains which do not satisfy this condition and hence this corollary does not follow from their results. In fact, it is unlikely that it follows from their techniques since Hölder domains are essentially more complicated domains.

Proof. By [GP] Lemma 2.1, we have that

$$
\log \frac{\delta_{D}\left(x_{0}\right)}{\delta_{D}(x)} \leq k_{D}\left(x_{0}, x\right)
$$

so $\left\{Q \in W \mid d(Q)=2^{-j}\right\} \subset\left\{x \in D \mid k_{D}\left(x_{0}, x\right) \gtrsim j\right\}$. The result now follows from Theorem 2 .

The next corollary concerns the Minkowski dimension of the boundary of a Hölder domain. For the definition of the Minkowski dimension of a set in $R^{n}$, see $[\mathrm{MV}]$ where it is shown that the Hausdorff dimension of a set is less than or equal to its Minkowski dimension.

Corollary 2. Let $D \subset R^{n}$ be an $\alpha$-Hölder domain. There is a constant $C>0$ depending only on the dimension $n$ such that the Minkowski dimension of the boundary of $D$ satisfies

$$
\operatorname{dim}_{M}(\partial D) \leq n-C \alpha^{n+1} .
$$

Proof. A Hölder domain is bounded, see [GM] or [SS2], and by Corollary 4 [SS2], the Euclidean measure of its boundary is 0. Thus we may apply Theorem 3.12 [MV] and Corollary 1 to conclude that $\operatorname{dim}_{M}(\partial D) \leq n-\beta$, where $\beta \gtrsim \alpha^{n+1}$ is the constant from Corollary 1. This completes the proof.

Remark 1. This result can also be established using condition (c) of Theorem 2 and the method of proof of Corollary 2 [SS2].

Remark 2. Jones and Makarov have recently established this result for simply connected planar domains $[\mathrm{M}]$.

The next corollaries concern the Poincaré inequality on a domain in $R^{n}$; see the introduction for definitions and notation. In [SS2] it was established that if $D$ is a Hölder domain, then $D$ is a $p$-Poincaré domain for $p \geq n$. We now are able to improve this bound on $p$ when $D$ is an $\alpha$-Hölder domain. 
Corollary 3. Let $D \subset R^{n}$ be an $\alpha$-Hölder domain. There is a constant $C>$ 0 depending only on the dimension $n$ such that $M_{p}(D)<\infty$ for $p>n-C \alpha^{n+2}$.

Proof. Since $D$ is an $\alpha$-Hölder domain, $D$ is bounded and (3.2) holds with $\beta \gtrsim \alpha^{n+1}$. Thus we may apply Theorem 7.12 of $[\mathrm{H}]$ to conclude that $M_{p}(D)<\infty$ for $p>n-\alpha \cdot \beta$. This finishes the proof.

Remark. An alternate proof of Corollary 3 can be based on Theorem 2 (a) implies (b) and Theorem 9 of [SS2].

In [SS2, Theorem 1] it was established that if $D \subset \mathbf{C}$ is the image of the unit disk in the complex plane under a Hölder continuous Riemann mapping function, then $M_{2}(D)<\infty$. Theorem 1 allows an extension of this to other Riemann mapping functions.

Corollary 4. Let $f$ be analytic and univalent in the unit disk. There is a positive constant $c$ such that if

$$
(1-|z|)\left|f^{\prime}(z)\right| \lesssim \exp \left(-c\left(\log \frac{1}{1-|z|}\right)^{2 / 3}\left(\log \log \frac{1}{1-|z|}\right)^{1 / 3}\right)
$$

for $|z|<1$, then $M_{2}(D)<\infty$, where $D=f(\{z \in \mathbf{C}|| z \mid<1\})$.

Proof. Let $\psi(t)=\exp \left(-c(\log (1 / t))^{2 / 3}(\log \log (1 / t))^{1 / 3}\right), 0<t<1$. Notice that $\int_{0}^{1}(\psi(t) / t) d t<\infty$, and so (3.3) implies that $D$ is bounded. Thus $m(D)<$ $\infty$, as is necessary for $D$ to be a Poincaré domain. By [SS2, Theorem 9], it is sufficient to show that

$$
\int_{D} k_{D}(f(0), x) d x<\infty
$$

The Koebe Distortion Theorem implies that $(1-|z|)\left|f^{\prime}(z)\right| \approx \delta_{D}(f(z))$, and furthermore that the hyperbolic metric, $\rho_{D}$, on $D$ satisfies

$$
\frac{1}{2} \rho_{D}\left(x_{1}, x_{2}\right) \leq k_{D}\left(x_{1}, x_{2}\right) \leq 2 \rho_{D}\left(x_{1}, x_{2}\right) .
$$

See Corollary 1.4 in [P] or [SS1]. Thus (3.3) can be restated as

$$
\begin{aligned}
\delta_{D}(f(z)) & \lesssim \psi(1-|z|) \leq \psi\left(2 \exp \left\{-\frac{k_{D}(f(0), f(z))}{2}\right\}\right) \\
& \lesssim \exp \left\{-a\left(\frac{\log k_{D}(f(0), f(z))}{k_{D}(f(0), f(z))}\right)^{1 / 3} \cdot k_{D}(f(0), f(z))\right\}
\end{aligned}
$$

where $a \approx c$. 
Now let $W=\{Q\}$ be a Whitney decomposition of $D$, and define $D_{j}$ as in Section 2 with $x_{0}=f(0)$. We assume without loss of generality that $\delta_{D}\left(x_{0}\right)=1$. We have shown that, for sufficiently large $j$,

$$
\delta_{D}(f(z)) \leq \exp \left(-\frac{a}{2}\left(\frac{\log j}{j}\right)^{1 / 3} \cdot j\right), \quad f(z) \in D_{i} \backslash D_{i-1} .
$$

Using this and Theorem 1, we get that

$$
\begin{aligned}
m\left(D_{j} \backslash D_{j-1}\right) & =\sum_{\substack{d(Q) \leq \exp \left(-(a / 2)(\log j / j)^{1 / 3} \cdot j\right) \\
Q \subset D_{j} \backslash D_{j-1}}} m(Q) \\
& \leq c_{3} d(D)^{n} \exp \left(-c_{4} \frac{a^{3}}{8} \frac{\log j}{j} \cdot j\right)=O\left(j^{-b}\right),
\end{aligned}
$$

where $b \approx a^{3} \approx c^{3}$. Thus if $c$ is sufficiently large,

$$
\int_{D} k_{D}\left(x_{0}, x\right) d x \lesssim \sum_{j=1}^{\infty} j \cdot m\left(D_{j} \backslash D_{j-1}\right)<\infty .
$$

This establishes (3.4) and completes the proof.

\section{Averaging domains}

Let $\varphi$ be a continuous increasing convex function on $[0, \infty)$ with $\varphi(0)=0$ and let $D$ be a domain, with $m(D)<\infty$. Following the treatment of Orlicz spaces given in Chapter VIII of [A], we define the Orlicz norm

$$
\|u\|_{L_{\varphi}(D)}=\inf \left\{k>0 \mid \frac{1}{m(D)} \int_{D} \varphi\left(\frac{|u(x)|}{k}\right) d x \leq 1\right\}
$$

for any measurable function $u$ on $D$. It follows that

$$
\frac{1}{m(D)} \int_{D} \varphi\left(\frac{|u(x)|}{\|u\|_{L_{\varphi}(D)}}\right) d x \leq 1
$$

whenever $\|u\|_{L_{\varphi}(D)}$ is finite.

Definition. A domain $D$, with $m(D)<\infty$, is a $\varphi$-averaging domain if there exists $M<\infty$ so that

$$
\left\|u-u_{D}\right\|_{L_{\varphi}(D)} \leq M \sup _{B \subset D}\left\|u-u_{B}\right\|_{L_{\varphi}(B)}
$$

whenever $u$ is an integrable function on $D$ and the supremum ranges over all balls $B$ contained in $D$. 
Remark 1. The use of the family of balls is not essential in the above definition. The following theorem is also valid if balls are replaced by cubes or more generally by the dilates of a bounded open set.

Remark 2. This definition with $L_{\varphi}(D)$ replaced by the $L^{p}(D)$ spaces, with $1 \leq p<\infty$, is given by Staples in $[\mathrm{S}]$, where the theorem below is proved in that setting. This is the special case $\varphi(t)=t^{p}$ in the following theorem.

Theorem 3. Suppose that $\varphi$ is as above and that $\varphi(t) \leq e^{b t}$ for some $0 \leq b<\infty$ and all $t \geq 1$. A domain $D$ is a $\varphi$-averaging domain if and only if

$$
\int_{D} \varphi\left(\varepsilon k_{D}\left(x_{0}, x\right)\right) d x<\infty
$$

for some $\varepsilon>0$ and $x_{0} \in D$.

Proof. Suppose that $D$ is a domain which satisfies (4.3). Then by the dominated convergence theorem,

$$
\lim _{\tau \rightarrow 0} \frac{1}{m(D)} \int_{D} \varphi\left(\tau k_{D}\left(x_{0}, x\right)\right) d x=0 .
$$

Let $u$ be an integrable function satisfying $\sup _{B}\left\|u-u_{B}\right\|_{L_{\varphi}(B)}<\infty$. Then by Jensen's inequality and (4.1) the $\operatorname{BMO}(D)$ norm of $u$ satisfies

$$
\|u\|_{*}=\sup _{B \subset D} \frac{1}{m(B)} \int_{B}\left|u-u_{B}\right| d x \leq \varphi^{-1}(1) \sup _{B \subset D}\left\|u-u_{B}\right\|_{L_{\varphi}(B)} .
$$

In addition, we see that the convexity of $\varphi$ yields a variant of the familiar BMOinequality, namely,

$$
\frac{1}{m(D)} \int_{D} \varphi\left(a\left|u-u_{D}\right|\right) d x \leq \frac{1}{m(D)} \int_{D} \varphi(2 a|u-c|) d x
$$

for any constant $c$ and any positive constant $a$.

Let $W=\{Q\}$ be a Whitney decomposition of $D$ into cubes. We may assume that each cube $Q \in W$ is contained in a ball $B_{Q}$, with $Q \subset B_{Q} \subset D$ and $m\left(B_{Q}\right) \lesssim m(Q)$. Using the techniques in Lemma 2.11 in $[\mathrm{S}]$ we see that

$$
\left|u_{B_{Q}}-u_{B_{0}}\right| \lesssim k_{D}\left(x_{0}, x_{Q}\right)\|u\|_{*}
$$

where we assume that $x_{0}$ is the center of $Q_{0} \in W, x_{Q}$ is the center of $B_{Q}$ and $B_{0}=B_{Q_{0}}$. 
Define $a$ by $a^{-1}=\sup _{B}\left\|u-u_{B}\right\|_{L_{\varphi}(B)}$ which we assume to be positive and let $\tau$ be a small positive number. By (4.6) we obtain the first inequality below:

$$
\begin{aligned}
\frac{1}{m(D)} \int_{D} \varphi\left(\frac{\tau a}{2}\left|u-u_{D}\right|\right) d x & \leq \frac{1}{m(D)} \int_{D} \varphi\left(\tau a\left|u-u_{B_{0}}\right|\right) d x \\
& \leq \sum_{Q} \frac{1}{m(D)} \int_{Q} \varphi\left(\tau a\left|u-u_{B_{Q}}\right|+\tau a\left|u_{B_{Q}}-u_{B_{0}}\right|\right) d x .
\end{aligned}
$$

We assume that $\tau$ is small enough so that the convexity of $\varphi,(4.5)$ and (4.7) imply that

$$
\begin{aligned}
\varphi\left(\tau a\left|u-u_{B_{Q}}\right|+\tau a\left|u_{B_{Q}}-u_{B_{0}}\right|\right) & \leq \varphi\left(\tau a\left|u-u_{B_{Q}}\right|+\tau c_{1} k_{D}\left(x_{0}, x_{Q}\right)\right) \\
& \leq \varphi\left(\tau a\left|u-u_{B_{Q}}\right|+(1-\tau) \tau c_{2} \inf _{x \in Q} k_{D}\left(x_{0}, x\right)\right) \\
& \leq \tau \varphi\left(a\left|u-u_{B_{Q}}\right|\right)+(1-\tau) \inf _{x \in Q} \varphi\left(\tau_{0} k_{D}\left(x_{0}, x\right)\right)
\end{aligned}
$$

where $\tau_{0}=\tau c_{2}$. Hence by (4.1), (4.4) and the properties of Whitney decompositions we get that

$$
\begin{aligned}
\frac{1}{m(D)} \int_{D} \varphi\left(\frac{1}{2} \tau a\left|u-u_{D}\right|\right) d x \leq \sum_{Q} & \left\{\frac{\tau}{m(D)} \int_{B_{Q}} \varphi\left(\frac{\left|u-u_{B_{Q}}\right|}{\left\|u-u_{B_{Q}}\right\|_{L_{\varphi}\left(B_{Q}\right)}}\right) d x\right. \\
& \left.+\frac{1}{m(D)} \int_{Q} \varphi\left(\tau_{0} k_{D}\left(x_{0}, x\right)\right) d x\right\} \\
\leq \tau c & +\frac{1}{m(D)} \int_{D} \varphi\left(\tau_{0} k_{D}\left(x_{0}, x\right)\right) d x \leq 1
\end{aligned}
$$

provided $\tau$ is sufficiently small. Thus,

$$
\left\|u-u_{D}\right\|_{L_{\varphi}(D)} \leq\left(\frac{1}{2} \tau a\right)^{-1}=\frac{2}{\tau} \sup _{B \subset D}\left\|u-u_{B}\right\|_{L_{\varphi}(B)}
$$

which proves that $D$ is a $\varphi$-averaging domain.

Conversely, we now assume that $D$ is a $\varphi$-averaging domain. Put $u(x)=$ $k_{D}\left(x_{0}, x\right)$. If $B \subset D$ is a ball of radius $r$ and center $x_{B}$, then we have by (4.6) that

$$
\begin{aligned}
\frac{1}{m(B)} \int_{B} \varphi\left(a\left|u-u_{B}\right|\right) d x & \leq \frac{1}{m(B)} \int_{B} \varphi\left(2 a\left|k_{D}\left(x_{0}, x\right)-k_{D}\left(x_{0}, x_{B}\right)\right|\right) d x \\
& \lesssim \frac{1}{r^{n}} \int_{|x| \leq r} \exp \left(2 a b \log \frac{r}{r-|x|}\right) d x \\
& =\int_{|x| \leq 1} \exp \left(2 a b \log \frac{1}{1-|x|}\right) d x \leq 1
\end{aligned}
$$


provided $a$ is sufficiently small. Hence, $\sup _{B \subset D}\left\|u-u_{B}\right\|_{L_{\varphi}(B)} \leq a^{-1}<\infty$.

Put $u_{j}(x)=\min (u(x), j)$. By a straightforward argument, we see from the above that

$$
\sup _{B \subset D}\left\|u_{j}-\left(u_{j}\right)_{B}\right\|_{L_{\varphi}(B)} \leq a^{-1}
$$

holds for $u_{j}$, all $j>0$, provided we make $a$ smaller. Since $k\left(x_{0}, x\right) \lesssim 1$ on $B_{0}$ we have by (4.2), that

$$
\begin{aligned}
\frac{1}{m(D)} \int_{D} u_{j} d x & \lesssim \frac{1}{m\left(B_{0}\right)} \int_{B_{0}}\left|u_{j}-\left(u_{j}\right)_{D}\right| d x+1 \\
& \leq \frac{m(D)}{m\left(B_{0}\right)} \frac{1}{m(D)} \int_{D}\left|u_{j}-\left(u_{j}\right)_{D}\right| d x+1 \\
& \leq \frac{m(D)}{m\left(B_{0}\right)} \varphi^{-1}(1)\left\|u_{j}-\left(u_{j}\right)_{D}\right\|_{L_{\varphi}(D)}+1 \\
& \leq \frac{m(D)}{m\left(B_{0}\right)} \varphi^{-1}(1) M a^{-1}+1 .
\end{aligned}
$$

Hence $k_{D}$ is integrable on $D$, since the right hand side is independent of $j$. We may therefore apply (4.2) and the above estimate for $\sup _{B}\left\|u-u_{B}\right\|_{L_{\varphi}(B)}$ to conclude that $\left\|u-u_{D}\right\|_{L_{\varphi}(D)} \leq M a^{-1}$.

Finally, we have

$$
\begin{aligned}
\int_{D} \varphi\left(\varepsilon k_{D}\left(x_{0}, x\right)\right) d x & \leq \int_{D} \varphi\left(\varepsilon\left|u-u_{D}\right|+\varepsilon\left|u_{D}\right|\right) d x \\
& \leq \frac{1}{2} \int_{D} \varphi\left(2 \varepsilon\left|u-u_{D}\right|\right) d x+\frac{1}{2} m(D) \varphi\left(2 \varepsilon\left|u_{D}\right|\right)<\infty
\end{aligned}
$$

provided $\varepsilon$ is small enough. This completes the proof.

Theorem 4. Let $\varphi(t)=e^{t}-1$. A domain $D$ is a $\varphi$-averaging domain if and only if $D$ is a Hölder domain.

Proof. If $D$ is a Hölder domain then $k_{D}$ is exponentially integrable by Theorem 2 and hence (4.3) holds and $D$ is a $\varphi$-averaging domain. Conversely, if $D$ is a $\varphi$-averaging domain, then by Theorem 3 there is a $\tau>0$ such that

$$
\int_{B} \exp \left(\tau k_{D}\left(x_{0}, x\right)\right) d x \leq 1+m(B)
$$

for all balls $B \subset D$. But this clearly implies that $D$ is a Hölder domain.

Lastly, we have the following generalization of the well known John-Nirenberg Theorem for cubes; see [JN]. 
Corollary 5. A necessary and sufficient condition that a domain $D$ be a Hölder domain is that there exists a $\tau>0$ such that the inequality

$$
\frac{1}{m(D)} \int_{D} \exp \left(\frac{\tau\left|u-u_{D}\right|}{\|u\|_{*}}\right) d x \leq 2
$$

holds for all $u \in \operatorname{BMO}(D)$.

Proof. Let $D$ be a Hölder domain and $u \in \operatorname{BMO}(D)$. By [S], we can replace the family of balls used in our definition of $\operatorname{BMO}(D)$ by the family of cubes contained in $D$. Thus, by the John-Nirenberg Theorem, there is a $\tau>0$ such that

$$
\frac{1}{m(Q)} \int_{Q} \exp \left(\frac{\tau\left|u-u_{D}\right|}{\|u\|_{*}}\right) d x \leq 2
$$

holds for any cube $Q \subset D$ and $u \in \operatorname{BMO}(D)$. Let $\varphi$ be as in Theorem 4, so that we have

$$
\sup _{Q \subset D}\left\|u-u_{Q}\right\|_{L_{\varphi(Q)}} \leq \tau^{-1}\|u\|_{*}
$$

and hence by Remark 1 (following (4.2)) and Theorem 4 we see that (4.8) holds for a smaller value of $\tau$.

Conversely, if (4.8) holds, then the inequality $\left\|u-u_{D}\right\|_{L_{\varphi(D)}} \leq \tau^{-1}\|u\|_{*}$ is valid for all $u \in \operatorname{BMO}(D)$. Trivially, $\|u\|_{*}$ is dominated by $\sup _{Q \subset D}\left\|u-u_{Q}\right\|_{L_{\varphi(D)}}$ and hence by Theorem 4 and Remark $1, D$ is a Hölder domain.

\section{References}

[A] Adams, Robert A.: Sobolev spaces. - Academic Press, New York, 1975.

[BP] Becker, J., and C. Pommerenke: Hölder continuity of conformal mappings and nonquasiconformal Jordan curves. - Comment. Math. Helv. 57, 1982, 221-225.

[C1] Carleson, L.: On convergence and growth of partial sums of Fourier series. - Acta Math. $116,1966,135-157$.

[C2] Carleson, L.: Two remarks on $H^{1}$ and BMO. - Adv. in Math. 22, 1976 269-277.

[CJ] Carleson, L., and P. Jones: On coefficient problems for univalent functions and conformal dimension. - Preprint.

[G] Garnett, J.B.: Bounded analytic functions. - Academic Press, New York, 1981.

[GM] Gehring, F.W., and O. MARTio: Lipschitz classes and quasiconformal mappings. - Ann. Acad. Sci. Fenn. Ser. A I Math. 10, 1985, 203-219.

[GO] Gehring, F.W., and B.G. OsGood: Uniform domains and the quasi-hyperbolic metric. - J. Analyse Math. 36, 1979, 50-74.

[GP] Gehring, F.W., and B.P. PalKa: Quasiconformally homogeneous domains. - J. Analyse Math. 30, 1976, 172-199.

[H] HURRI, R.: Poincaré domains in $R^{n}$. - Ann. Acad. Sci. Fenn. Ser. A I Math. Dissertationes $71,1988,1-42$. 
[JN] John, F., and L. Nirenberg: On functions of bounded mean oscillation. - Comm. Pure Appl. Math. 14, 1961, 415-426.

[M] Makarov, N.G.: Private communication.

[MV] Martio, O., and M. Vuorinen: Whitney cubes, $p$-capacity, and Minkowski content. Exposition. Math. 5, 1987, 17-40.

[P] Pommerenke, C.: Univalent functions. - Vandenhoeck and Ruprecht, Göttingen, 1975.

[SS1] Smith, W., and D.A. Stegenga: A geometric characterization of Hölder domains. - J. London Math. Soc. 35, 1987, 471-480.

[SS2] Smith, W., and D.A. Stegenga: Hölder domains and Poincaré domains. - Trans. Amer. Math. Soc. 319, 1990, 67-100.

[S] Staples, S.G.: $L^{p}$ averaging domains and the Poincaré inequality. - Ann. Acad. Sci. Fenn. Ser. A I Math. 14, 1989, 103-127.

[St] Stein, E.M.: Singular integrals and differentiability properties of functions. - Princeton University Press, Princeton, NJ, 1970.

[Z] Zygmund, A.: On certain lemmas of Marcinkiewicz and Carleson. - J. Approx. Theory $2,1969,249-257$.

Wayne Smith

University of Hawaii

Department of Mathematics

Honolulu, Hawaii 96822

U.S.A.

Received 21 December 1990
David A. Stegenga

University of Hawaii

Department of Mathematics

Honolulu, Hawaii 96822

U.S.A. 